\title{
Drawing a Line in the Sky: Astronomers Confront Satellite Threat
}

\author{
The astronomy community has released recommendations for mitigating \\ the impact of thousands of internet-providing satellites that companies \\ plan to launch in the coming years.
}

By Michael Schirber

$T$ he sky is changing. As many astronomers and backyard onlookers have noticed, a number of unfixed "stars" have recently appeared. These moving blips are Starlink satellites, launched by the SpaceX corporation with the aim of providing high-speed internet to remote regions of the planet. Currently, about 600 Starlink satellites are in orbit, but SpaceX-as well as other companies-plan to fly many thousands more. These so-called satellite constellations could have a severe impact on astronomical observations. The astronomy community has now released recommendations for both the satellite industry and sky observers that could reduce the chances that a satellite "photobombs" a telescope image.

Astronomers have had to deal with satellites for decades. Around 5000 satellites are currently in orbit. Most are faint and travel along well-defined orbits, so astronomers can usually time an observation to avoid them or use software to remove their light from an image. But the problem took on a new magnitude when SpaceX launched its first bevy of Starlink satellites in March 2019. Sunlight reflecting off the satellites made them visible to the naked eye, with some observers reporting a "string of pearls" marching across the sky. "The brightness of the SpaceX satellites was a surprise to the entire astronomical community," says Ralph Gaume, director of the astronomical sciences division at the National Science Foundation (NSF).

The satellites are so bright, in part, because they operate at 550 $\mathrm{km}$ above Earth. Most other communications satellites fly roughly $35,000 \mathrm{~km}$ above Earth in a geosynchronous orbit, but SpaceX chose low-Earth orbit to obtain a factor of 70 speedup in the time it takes internet signals to travel up to and back from the satellite. The company has approval for 12,000 satellites from the Federal Communications Commission, but it is looking to add 30,000 more. Other companies, such as Amazon and OneWeb, have announced plans to launch constellations of similar sizes. "An image with satellite trails happens occasionally now," says Jeff Hall from Lowell Observatory in Arizona. "But with plans for 100,000-plus satellites, it'll be happening almost all the time."

To address this looming predicament, the American Astronomical Society (AAS) and the NSF's National Optical-Infrared Astronomy Research Laboratory (NOIRLab) organized the first Satellite Constellation Workshop in June 2020. Over 250 astronomers and industry representatives participated. "Our two main goals were to figure out what the impacts are and then to look at what technical fixes, or mitigations, we might implement" explains Hall, who co-chaired the Workshop. The main takeaways from the meeting have now been released in a report.

Using preliminary observations of Starlink satellites and computer simulations, the Workshop committee predicted the potential ramifications of tens of thousands of low-Earth-orbit satellites. One model, for example, showed that a telescope taking a 30-s exposure of the Large Magellanic Cloud-a nearby dwarf galaxy-would almost always have at least one satellite 


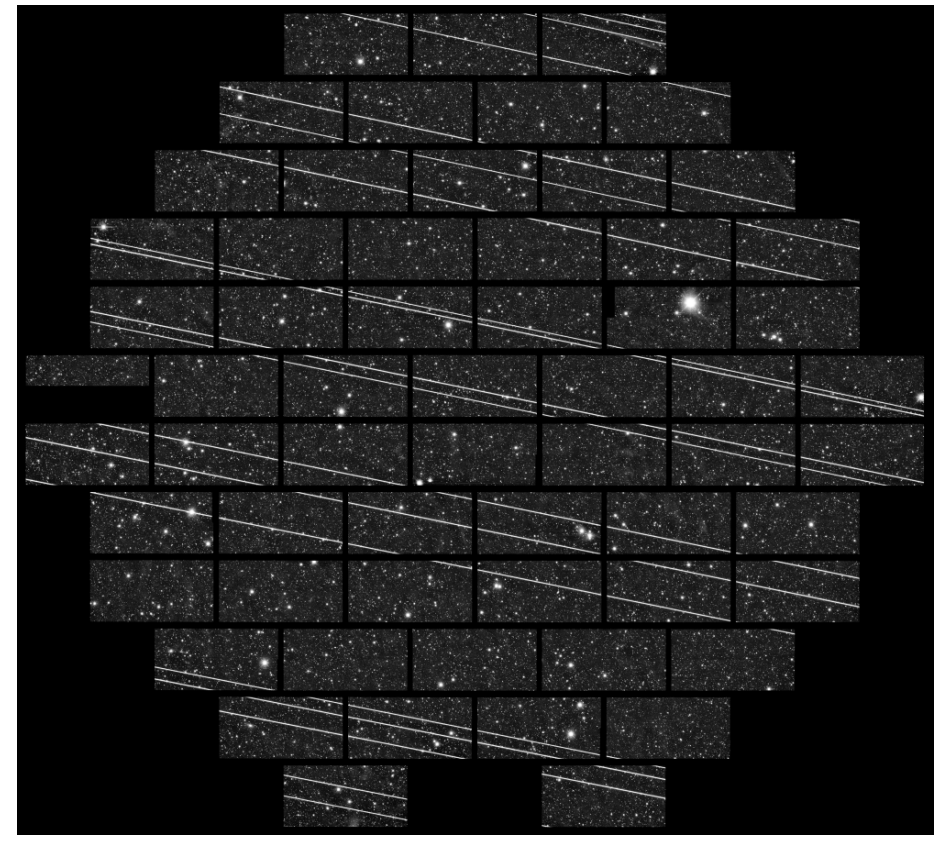

Trails from 19 Starlink satellites were captured in this composite image taken in November 2019 by a camera on the Blanco 4-m telescope at the Cerro Tololo Inter-American Observatory (CTIO) in Chile.

Credit: CTIO/NOIRLab/NSF/AURA/DECam DELVE Survey

pass in front of it. A December 2019 survey found that many observatories expect the coming constellations to impact their science objectives, but the one that will likely be most affected is the Vera Rubin Observatory being built in Chile. Its large field of view (the equivalent of 40 full moons) is sure to pick up satellite trails. Simulations with the observatory's detectors suggest that bright trails will cause an "electronic echo" that will be difficult to remove from the data, says Rubin's chief scientist Tony Tyson from the University of California, Davis. The corruption of such images could jeopardize the detection of faint transients, such as gravitational-wave counterparts and potentially hazardous asteroids.

The report outlined a number of mitigation strategies. One of the main recommendations is that satellites should fly below $600 \mathrm{~km}$. At this height, the satellites are bright during twilight hours, but they become nearly invisible in the middle of the night when they pass in the shadow of Earth. By contrast, satellites at $1200 \mathrm{~km}$-the operational orbit for some planned constellations-would be visible for most of the night. This higher (slower moving) orbit would also cause the objects to have a stronger cumulative effect on camera pixels. "That's really a dangerous place to be, in terms of impact on science," Tyson says. Another recommendation is to darken the satellites to a brightness of 7 th magnitude, which is about 2.5 times fainter than the limit of naked eye visibility. SpaceX has made efforts in this direction by using black paint and installing Sun-blocking visors on some of their satellites. NOIRLab's Lori Allen says that further observations are necessary to assess the efficacy of these design changes as well as other darkening strategies.

Hall says that SpaceX has been very proactive and that Amazon has also reached out to the astronomy community. But one of the big concerns is that future satellite operators might not care about the light pollution that they produce. "It's kind of a wild west up there," Hall says. Ensuring that the lights stay dimmed in our cosmic front porch will require government involvement. To get that ball rolling, the AAS and NSF are planning a second meeting in 2021 that will focus on policy initiatives.

Michael Schirber is a Corresponding Editor for Physics based in Lyon, France. 